\title{
ISOPERIMETRIC FUNCTIONS OF GROUPS ACTING ON $L_{\delta}$-SPACES
}

\author{
JON CORSON \\ University of Alabama, Tuscaloosa, Alabama 35487, USA \\ e-mail: jcorson@bama.ua.edu \\ and DOHYOUNG RYANG \\ Talladega College, Talladega, Alabama 35160, USA \\ e-mail: dryang@talladega.edu
}

(Received 9 December, 2005; revised 18 September, 2006; accepted 22 November, 2006)

\begin{abstract}
A finitely generated group acting properly, cocompactly, and by isometries on an $L_{\delta}$-metric space is finitely presented and has a sub-cubic isoperimetric function.
\end{abstract}

2000 Mathematics Subject Classification. 20F65.

1. Introduction. Recently the $L_{\delta}$-property has been introduced by Chatterji [2]. In [3], spaces with the $L_{\delta}$-property are shown to have applications to group $C^{*}$-algebras. This property is used to define $L_{\delta}$-groups, which are a generalization of hyperbolic groups. The precise definitions are given in Section 2.

Hyperbolic groups are characterized as the groups with a linear Dehn function [5]. Elder showed that if a Cayley graph $\Gamma(G, A)$ enjoys the $L_{\delta}$-property, then $G$ has a subcubic Dehn function [4]. This suggests the following question asked by I. Chatterji and K. Ruane (Albany conference talk, 2004): If a group $G$ acts properly, cocompactly, and by isometries on an $L_{\delta}$-space, then what is a bound for the Dehn function of $G$ ?

In this paper we give an answer to this question by showing that Elder's result generalizes to groups that are quasi-isometric to an $L_{\delta}$-metric space (Theorem 3.2). It should be noted that it is unknown whether or not such a group always admits a finite generating set for which the Cayley graph is an $L_{\delta}$-metric space.

2. Preliminary results. Let $G$ be a group with finite presentation $\langle A \mid R\rangle$ and let $\Delta$ be a connected graph in $\mathbb{R}^{2}$ whose edges are oriented and labeled by elements in $A$. The graph $\Delta$ is said to be a van Kampen Diagram for $w \in A^{*}$ if reading the labels around the boundary of $\Delta$ gives $w$, and reading the labels on the boundary of each region gives a relator in $R^{ \pm}$. A word $w$ has a van Kampen diagram if and only if $\bar{w}=1$, and the area $\mathcal{A}(w)$ is equal to the minimum number of regions in a van Kampen diagram for $w$.

The function $\mathcal{D}(n)=\max \left\{\mathcal{A}(w):|w|_{A} \leq n, \bar{w}=1\right\}$ is called the Dehn function for the group presentation $\langle A \mid R\rangle$. An isoperimetric function for this presentation is any function satisfying $\mathcal{D}(n) \leq f(n)$. To make the Dehn function independent of the presentation, we define an equivalence relation on functions. The notation $f \preceq g$ means that there are positive constants $A, B, C, D, E$ such that $f(n) \leq A g(B n+C)+D n+E$. 
Two functions $f$ and $g$ are said to be equivalent, denoted $f \sim g$, if $f \preceq g \preceq f$. If two finitely presented groups $G$ and $H$ are quasi-isometric, then their Dehn functions are equivalent; see for example [7]. In particular, the Dehn function of $G$ is independent of its presentations up to this equivalence.

Let $(X, d)$ be a metric space and let $\delta \geq 0$ be a constant. A finite sequence $\left(x_{1}, x_{2}, \ldots, x_{n}\right)$ of points $x_{1}, x_{2}, \ldots, x_{n}$ in $X$ is said to be a $\delta$-path, if $d\left(x_{1}, x_{2}\right)+$ $d\left(x_{2}, x_{3}\right)+\ldots+d\left(x_{n-1}, x_{n}\right) \leq d\left(x_{1}, x_{n}\right)+\delta$. Choose $x, y, z \in X$. If there exists a point $t \in X$ so that the paths $(x, t, y),(y, t, z)$, and $(z, t, x)$ are all $\delta$-paths, $t$ is called a $\delta$-center for a triple $x, y, z$. We say that a geodesic metric space $(X, d)$ has the $L_{\delta}$-property and call it an $L_{\delta}$-metric space, or an $L_{\delta}$-space for short, if every triple $x, y, z \in X$ has a $\delta$-center in $X$. Of course the $L_{\delta}$-property makes sense for metric spaces in general, but here we are only interested in geodesic metric spaces.

DEFINITION 2.1 ( $L_{\delta}$-group). An $L_{\delta}$-group is a finitely generated group $G$ that acts properly, cocompactly, and by isometries on an $L_{\delta}$-space for a constant $\delta \geq 0$.

Next we introduce the Rips graph of a geodesic metric space $(X, d)$. Let $s>0$ be a constant. Construct a metric graph $\Gamma_{s}(X)$ by requiring that $\mathcal{V}\left(\Gamma_{s}(X)\right)=X$ and $[x, y] \in \mathcal{E}\left(\Gamma_{s}(X)\right)$ if and only if $0<d(x, y) \leq s$. By $d_{s}$ denote the path metric obtained by making each edge isometric to the unit interval $[0,1]$. If $\gamma$ is an edge path in $\Gamma_{s}(X)$, then $\ell(\gamma)$ is the number of edges in $\gamma$. That is, $\Gamma_{s}(X)$ is the 1-skeleton of the Rips complex for $(X, d)$ with parameter $s$. It is easy to see that $\left(\Gamma_{s}(X), d_{s}\right)$ is a geodesic space. Moreover, the Rips graph is a generalization of the Cayley graph: Taking $(X, d)=\left(G, d_{A}\right)$, where $G$ is a group generated by a finite set $A$ and $d_{A}$ is the corresponding word metric, $\Gamma_{S}(X)$ is the Cayley graph $\Gamma(G, A)$.

LEMMA 2.2. Let $(X, d)$ be a geodesic space and $\Gamma_{s}(X)$ be its associated Rips graph. Then for all $s \geq 1$,

(1) $\frac{1}{s} d(x, y) \leq d_{s}(x, y)<\frac{1}{s} d(x, y)+1$ for all $x, y \in X$,

(2) $(X, d)$ and $\left(\Gamma_{s}(X), d_{s}\right)$ are quasi-isometric.

Proof. (1) For the first inequality, let $d_{s}(x, y)=n$. Then there is a geodesic path $\gamma=\left[x_{0}, x_{1}\right]\left[x_{1}, x_{2}\right] \ldots\left[x_{n-1}, x_{n}\right]$ where $\gamma(0)=x_{0}=x, \gamma(1)=x_{n}=y$. Note that each $\left[x_{i}, x_{i+1}\right]$ is an edge in $\Gamma_{s}(X)$, i.e., $d_{s}\left(x_{i}, x_{i+1}\right)=1$ and $d\left(x_{i}, x_{i+1}\right) \leq s$. Thus

$$
d(x, y) \leq d\left(x_{0}, x_{1}\right)+d\left(x_{1}, x_{2}\right)+\ldots+d\left(x_{n-1}, x_{n}\right) \leq s \cdot n=s \cdot d_{s}(x, y) .
$$

For the second inequality, let $\gamma$ be a geodesic path from $x$ to $y$. Choose a partition $\mathcal{P}: t_{0}<t_{1}<\cdots<t_{n}$ on $[0,1]$ where $t_{0}=0, t_{n}=1, d\left(\gamma\left(t_{i-1}\right), \gamma\left(t_{i}\right)\right)=s$ for all $1 \leq i \leq$ $n-1$, and $0<d\left(\gamma\left(t_{n-1}\right), \gamma\left(t_{n}\right)\right) \leq s$. Let $x_{i}=\gamma\left(t_{i}\right), x=x_{0}$, and $y=x_{n}$. Then there is an edge path $\left[x_{0}, x_{1}\right]\left[x_{1}, x_{2}\right] \cdots\left[x_{n-1}, x_{n}\right]$ in $\Gamma_{s}(X)$ from $x$ to $y$. Thus,

$$
d_{s}(x, y) \leq \frac{1}{s} \sum_{i=1}^{n-1} d\left(x_{i-1}, x_{i}\right)+1 \leq \frac{1}{s} \ell(\gamma)+1=\frac{1}{s} d(x, y)+1 .
$$

(2) By the above fact (1), the identity $\iota:(X, d) \rightarrow\left(\Gamma_{s}(X), d_{s}\right)$ is a quasi-isometric embedding. And every point in $\Gamma_{s}(X)$ is less than one edge apart from some vertex in $X \subset \Gamma_{s}(X)$. 
It is an open question whether or not the $L_{\delta}$-property is invariant under quasiisometries. Nevertheless, in the next section we reduce to the case of a metric graph by way of the following lemma.

LEMMA 2.3. If $(X, d)$ is an $L_{\delta}$-space, then $\left(\Gamma_{s}(X), d_{s}\right)$ is an $L_{\delta^{\prime \prime}}$-space where $\delta^{\prime \prime}=\frac{\delta}{s}+6$ and $s \geq 1$.

Proof. First choose $x, y, z \in X \subset \Gamma_{s}(X)$ and let $t \in X$ be a $\delta$-center of the triple $x, y, z$ in $(X, d)$. By Lemma 2.2.(1),

$$
d_{s}(x, t)+d_{s}(t, y) \leq d_{s}(x, y)+\frac{\delta}{s}+2 .
$$

Also, $d_{s}(y, t)+d_{s}(t, z) \leq d_{s}(y, z)+\frac{\delta}{s}+2$ and $d_{s}(x, t)+d_{s}(t, z) \leq d_{s}(x, z)+\frac{\delta}{s}+2$. So $t \in X$ is a $\delta^{\prime}$-center of the triple $x, y, z$ in $\left(X, d_{s}\right)$, and hence $\left(X, d_{s}\right)$ is an $L_{\delta^{\prime}}$-space for $\delta^{\prime}=\frac{\delta}{s}+2$.

Now let $x, y, z$ be in $\Gamma_{s}(X)$. Choose $x^{\prime}, y^{\prime}, z^{\prime} \in X=\mathcal{V}\left(\Gamma_{s}(X)\right)$ such that $d_{s}\left(x, x^{\prime}\right)<$ $1, d_{s}\left(y, y^{\prime}\right)<1$, and $d_{s}\left(z, z^{\prime}\right)<1$. Let $t \in X \subset \Gamma_{s}(X)$ be a $\delta^{\prime}$-center for $x^{\prime}, y^{\prime}, z^{\prime}$ in $\left(X, d_{s}\right)$. A simple calculation shows that

$$
\begin{aligned}
d_{s}(x, t)+d_{s}(t, y) & \leq d_{s}\left(x, x^{\prime}\right)+d_{s}\left(x^{\prime}, t\right)+d_{s}\left(t, y^{\prime}\right)+d_{s}\left(y^{\prime}, y\right) \\
& \leq d_{s}\left(x^{\prime}, y^{\prime}\right)+\delta^{\prime}+2 \\
& \leq d_{s}\left(x^{\prime}, x\right)+d_{s}(x, y)+d_{s}\left(y, y^{\prime}\right)+\delta^{\prime}+2 \\
& \leq d_{s}(x, y)+\delta^{\prime}+4
\end{aligned}
$$

Similarly, $d_{s}(y, t)+d_{s}(t, z) \leq d_{s}(y, z)+\delta^{\prime}+4$ and $d_{s}(z, t)+d_{s}(t, x) \leq d_{s}(z, x)+\delta^{\prime}+4$. Take $\delta^{\prime \prime}=\delta^{\prime}+4=\frac{\delta}{s}+6$. Then $t \in \Gamma_{s}(X)$ is a $\delta^{\prime \prime}$-center for the triple $x, y, z \in \Gamma_{s}(X)$, and hence $\left(\Gamma_{s}(X), d_{s}\right)$ is an $L_{\delta^{\prime \prime}}$-space for $\delta^{\prime \prime}=\frac{\delta}{s}+6$.

3. Main result. We first observe a fact about polygons in $\mathbb{R}^{2}$. By a polygon in $\mathbb{R}^{2}$, we mean a simple closed curve consisting of a finite number of line segments, called edges. For each edge $e$ of a polygon $P$, let $H_{e}$ be the open half-plane on the side of the line through $e$ determined by a $P$-inward pointing normal vector to $e$. Define the convex core of $P$ by $\mathcal{C}(P)=\bigcap_{e \in \mathcal{E}(P)} H_{e}$. Being an intersection of half-planes, $\mathcal{C}(P)$ is convex, and in some bad cases it is empty.

Assume that $\mathcal{C}(P)$ is non-empty, and choose $c \in \mathcal{C}(P)$. Then for all $x \in P,[x, c] \cap$ $P=\{x\}$, where $[x, c]$ is a straight line segment. Note, in particular, that if $P$ is a convex polygon, then $\mathcal{C}(P)$ is the inside of $P$. The following lemma is obvious and easy to prove.

Lemma 3.1. Suppose that $P$ is a polygon in $\mathbb{R}^{2}$ with non-empty convex core and let $x, y, z$ be distinct vertices of $P$. If $c \in \mathcal{C}(P)$, then the three line segments $[x, c],[y, c]$, and $[z, c]$ subdivide $P$ into three polygons, each with non-empty convex core.

Let $(X, d)$ be an $L_{\delta}$-space and $\Gamma_{s}(X)$ be the associated Rips graph with parameter $s \geq 1$. We now give a procedure for constructing a sequence of planar combinatorial graphs and combinatorial maps to $\Gamma_{s}(X)$ which we use in the proof of the main theorem. This is similar to the procedure used by Elder [4] in a Cayley graph. By Lemma 3.1, these combinatorial graphs can be constructed by vertices and straight edges. 
Let a convex $n$-gon $\Delta_{0}$ in $\mathbb{R}^{2}$ with vertices $v_{0}, v_{1}, \ldots, v_{n-1}$ in this order and a combinatorial map $\varphi_{0}: \Delta_{0} \rightarrow \Gamma_{s}(X)$ be given. Put $x_{i}=\varphi_{0}\left(v_{i}\right)$. Note that $d\left(x_{i}, x_{i+1}\right) \leq s$ since $\left[x_{i}, x_{i+1}\right]$ is an edge in $\Gamma_{s}(X)$.

Construct $\Delta_{1}$. If $n>3 \delta^{\prime \prime}+8$, then we subdivide $\Delta_{0}$ as follows: Let $p=\left\lfloor\frac{n}{3}\right\rfloor$ and $q=\left\lfloor\frac{2 n}{3}\right\rfloor$, where \lfloor\rfloor is the greatest integer function. Then the three vertices $v_{0}, v_{p}, v_{q}$ subdivide $\Delta_{0}$ into three sub-paths, each of edge length less than or equal to $\left\lfloor\frac{n}{3}\right\rfloor+1$. Let $t$ be a $\delta$-center for $x_{0}, x_{p}, x_{q}$ in $(X, d)$. Then by Lemma 2.3 and its proof, $t$ is also a $\delta^{\prime \prime}$-center for $x_{0}, x_{p}, x_{q}$ in $\left(\Gamma_{s}(X), d_{s}\right)$, where $\delta^{\prime \prime}=\frac{\delta}{s}+6$. Since $\Delta_{0}$ is convex, its convex core is non-empty. Choose a point $c \in \mathcal{C}\left(\Delta_{0}\right)$ so that the three line segments $\left[v_{0}, c\right],\left[v_{p}, c\right]$ and $\left[v_{q}, c\right]$ intersect only at $c$. Then $\Delta_{1}$ has 3 regions.

Define a $\operatorname{map} \varphi_{1}: \Delta_{1} \rightarrow \Gamma_{s}(X)$ by requiring that $\left.\varphi_{1}\right|_{\Delta_{0}}=\varphi_{0}, \varphi_{1}(c)=t$, and $\varphi_{1}\left[c, v_{i}\right]$ is a geodesic path in $\Gamma_{s}(X)$ from $t=\varphi_{1}(c)$ to $x_{i}=\varphi_{1}\left(v_{i}\right)$ where $i=0, p, q$. Subdivide each of $\left[v_{0}, c\right],\left[v_{p}, c\right]$, and $\left[v_{q}, c\right]$ so that $\varphi_{1}$ maps them combinatorially onto their images in $\Gamma_{s}(X)$. Define the combinatorial length $\ell(\gamma)$ of a path $\gamma$ in $\Delta_{1}$ to be the number of edges in $\gamma$. Then

$$
\ell\left(\left[v_{p}, c\right]\right)+\ell\left(\left[c, v_{q}\right]\right)=d_{s}\left(x_{p}, t\right)+d_{s}\left(t, x_{q}\right) \leq d_{s}\left(x_{p}, x_{q}\right)+\delta^{\prime \prime} \leq \frac{n}{3}+1+\delta^{\prime \prime} .
$$

Similarly, $\ell\left(\left[v_{0}, c\right]\right)+\ell\left(\left[c, v_{p}\right]\right) \leq \frac{n}{3}+1+\delta^{\prime \prime}$ and $\ell\left(\left[v_{0}, c\right]\right)+\ell\left(\left[c, v_{q}\right]\right) \leq \frac{n}{3}+1+\delta^{\prime \prime}$. So the combinatorial perimeter of each region of $\Delta_{1}$ is bounded by

$$
\frac{n}{3}+1+\frac{n}{3}+1+\delta^{\prime \prime}=\frac{2 n}{3}+2+\delta^{\prime \prime}
$$

Recall that $n>3 \delta^{\prime \prime}+8$ or $\delta^{\prime \prime}<\frac{n-8}{3}$. Thus it is shorter than $\frac{2 n}{3}+2+\frac{n-8}{3}=n-\frac{2}{3}<n$. That is, the combinatorial perimeter of each new region in $\Delta_{1}$ is strictly shorter than the combinatorial perimeter of $\Delta_{0}$.

Repeat this trisection process on each region in $\Delta_{1}$ whose perimeter is greater than $3 \delta^{\prime \prime}+8$ to construct $\Delta_{2}$ and $\varphi_{2}: \Delta_{2} \rightarrow \Gamma_{s}(X)$. Thus, the number of regions in $\Delta_{2}$ is less than or equal to $3^{2}$ and the combinatorial perimeter of each new region in $\Delta_{2}$ is bounded by

$$
\frac{2}{3}\left(\frac{2 n}{3}+2+\delta^{\prime \prime}\right)+2+\delta^{\prime \prime}=\left(\frac{2}{3}\right)^{2} n+\frac{2}{3}\left(2+\delta^{\prime \prime}\right)+\left(2+\delta^{\prime \prime}\right) .
$$

Choose $k$ so that $\left(\frac{3}{2}\right)^{k} \leq n<\left(\frac{3}{2}\right)^{k+1}$. After $k$ iterations, we have $\Delta_{k}$ and $\varphi_{k}: \Delta_{k} \rightarrow$ $\Gamma_{s}(X)$. Thus $\Delta_{k}$ has at most $3^{k}$ regions, and the combinatorial perimeter of each region in $\Delta_{k}$ is at most

$$
\left(\frac{2}{3}\right)^{k} n+\left(\frac{2}{3}\right)^{k-1}\left(\delta^{\prime \prime}+2\right)+\cdots+\frac{2}{3}\left(2+\delta^{\prime \prime}\right)+\left(2+\delta^{\prime \prime}\right)
$$

which is bounded by $\left(\frac{2}{3}\right)^{k}\left(\frac{3}{2}\right)^{k+1}+\frac{\delta^{\prime \prime}+2}{1-\frac{2}{3}}<3 \delta^{\prime \prime}+8$. In particular, our procedure terminates after at most $k$ steps.

THEOREM 3.2. If a finitely generated group $G$ is quasi-isometric to an $L_{\delta}$-space for some $\delta \geq 0$, then $G$ is finitely presented and has a sub-cubic Dehn function.

Proof. Let $A$ be a finite generating set for $G$ which is inverse closed and use the word metric $d_{A}$ for $G$. Suppose $\left(G, d_{A}\right)$ is quasi-isometric to an $L_{\delta}$-space $(X, d)$. Choose 
quasi-isometries $\alpha: G \rightarrow X$ and $\beta: X \rightarrow G$ such that for all $g \in G$ and $x \in X$, $d_{A}(g,(\beta \circ \alpha)(g)) \leq C$ and $d(x,(\alpha \circ \beta)(x)) \leq C$, where $C$ is a constant. We may assume that $\alpha$ and $\beta$ are both $(\lambda, \varepsilon)$-quasi-isometries with the same constants $\lambda \geq 1$ and $\varepsilon \geq 0$.

Choose $w$ in $A^{*}$ so that $w=a_{1} a_{2} \ldots a_{n}$ where $a_{i} \in A$ and $\bar{w}=1$. Let $g_{i}=\overline{a_{1} \ldots a_{i}} \in G$. We want to construct a van Kampen diagram for $w$. Start with a convex $n$-gon $\Delta_{0}$ in $\mathbb{R}^{2}$ with vertices $v_{0}, \ldots, v_{n-1}$ in this order.

Put $s=\lambda+\varepsilon$ and define $\varphi_{0}: \Delta_{0} \rightarrow \Gamma_{s}(X)$ by $\varphi_{0}\left(v_{i}\right)=\alpha\left(g_{i}\right)=x_{i}$, say. Note that $\left[x_{i}, x_{i+1}\right]$ is an edge in $\Gamma_{s}(X)$, since

$$
d\left(x_{i}, x_{i+1}\right)=d\left(\alpha\left(g_{i}\right), \alpha\left(g_{i+1}\right)\right) \leq \lambda d_{A}\left(g_{i}, g_{i+1}\right)+\varepsilon=\lambda+\varepsilon=s .
$$

So the map $\varphi_{0}$ is a closed path in $\Gamma_{s}(X)$ with combinatorial length $n$.

If $n=|w|_{A}$ is greater than $3 \delta^{\prime \prime}+8$, then trisect $\Delta_{0}$ to construct $\Delta_{1}$ and $\varphi_{1}: \Delta_{1} \rightarrow$ $\Gamma_{s}(X)$. Iterate the trisection process for each region whose combinatorial perimeter is greater than $3 \delta^{\prime \prime}+8$ until all regions have perimeter shorter than $3 \delta^{\prime \prime}+8$. Suppose this is achieved after $k$-iteration. Thus we have $\Delta_{k}$ and $\varphi_{k}: \Delta_{k} \rightarrow \Gamma_{s}(X)$, where $\Delta_{k}$ has at most $3^{k}$ regions and $\left(\frac{3}{2}\right)^{k} \leq n<\left(\frac{3}{2}\right)^{k+1}$.

In order to get an $n$-gon which is mapped to a closed path of $A$-length $n$ in $\Gamma(G, A)$, we inflate $\Delta_{k}$ a bit to form the graph $\Delta$. We put $n$ vertices on the outside of $\Delta_{k}$ labeled by $y_{0}, \ldots, y_{n-1}$ and put $2 n$ edges $\left[v_{i}, y_{i}\right]$ and $\left[y_{i}, y_{i+1}\right]$ where $i=0,1, \ldots, n-1 \bmod n$. Thus $\Delta$ has $n$ regions outside of $\Delta_{k}$.

Define a map $\varphi: \Delta \rightarrow \Gamma(G, A)$ as follows: (1) $\varphi$ is the composition $\mathcal{V}\left(\Delta_{k}\right) \stackrel{\varphi_{k}}{\longrightarrow}$ $X \stackrel{\beta}{\longrightarrow} G \hookrightarrow \Gamma(G, A)$; (2) $\varphi\left(y_{i}\right)=g_{i}$; and (3) $\varphi([u, v])$ is a geodesic path from $\varphi(u)$ to $\varphi(v)$ in $\Gamma(G, A)$, for $[u, v] \in \mathcal{E}(\Delta)$. Then $\varphi$ maps $\partial \Delta$ to a closed path labeled by $w$ in $\Gamma(G, A)$ of $A$-length $n$.

We now show that each region in $\Gamma(G, A)$ has a perimeter bounded by a constant. If $[u, v] \in \mathcal{E}\left(\Delta_{k}\right)$, then

$$
|\varphi([u, v])|_{A}=d_{A}\left(\left(\beta \circ \varphi_{k}\right)(u),\left(\beta \circ \varphi_{k}\right)(v)\right) \leq \lambda d\left(\varphi_{k}(u), \varphi_{k}(v)\right)+\varepsilon \leq \lambda s+\varepsilon .
$$

Recall that the combinatorial perimeter of each region of $\Delta_{k}$ is bounded by $3 \delta^{\prime \prime}+8$. So, for each region $D$ in $\Delta_{k}, \varphi(\partial D)$ is a closed path in $\Gamma(G, A)$ of length at most $\left(3 \delta^{\prime \prime}+8\right)(\lambda s+\varepsilon)$. And for each outer region $M$ in $\Delta \backslash \Delta_{k}, \varphi(\partial M)=$ $\varphi\left[v_{i}, v_{i+1}\right] \varphi\left[v_{i+1}, y_{i+1}\right] \varphi\left[y_{i+1}, y_{i}\right] \varphi\left[y_{i}, v_{i}\right]$ in $\Gamma(G, A)$, and

$$
d_{A}\left(\varphi\left(y_{i}\right), \varphi\left(y_{i+1}\right)\right)=1 ; d_{A}\left(\varphi\left(v_{i}\right), \varphi\left(y_{i}\right)\right) \leq C ; d_{A}\left(\varphi\left(v_{i}\right), \varphi\left(v_{i+1}\right)\right) \leq \lambda s+\varepsilon .
$$

So, $\varphi(\partial M)$ is the closed path in $\Gamma(G, A)$ of length at most $\lambda s+\varepsilon+2 C+1$.

Let $K=\max \left\{(\lambda s+\varepsilon)\left(3 \delta^{\prime \prime}+8\right), \lambda s+\varepsilon+2 C+1\right\}$. Then the perimeter of every region in $\Gamma(G, A)$ is bounded by $K$, whence $\left\{w \in A^{*} \mid \bar{w}=1\right.$ and $\left.|w|_{A} \leq K\right\}$ is a finite set of defining relators for $G$, and so $G$ is finitely presented.

The area $\mathcal{A}(w)$ is at most $3^{k}+n$. Remember $\left(\frac{3}{2}\right)^{k} \leq n$ or $k \leq \log _{1.5} n$. Hence

$$
\mathcal{A}(w) \leq 3^{k}+n \leq 3^{\log _{1.5} n}+n=n^{\log _{1.5} 3}+n \sim n^{\log _{1.5} 3} .
$$

If $|w|_{A}=n \leq 3 \delta^{\prime \prime}+8$, then $w$ is a relator so again $\mathcal{A}(w)=1 \leq n^{\log _{1.5} 3}$.

We obtain the following statement which is an answer to the question posed in the introduction. 
COROLLARY 3.3. If a group $G$ acts properly, cocompactly, and by isometries on an $L_{\delta}$-space for some $\delta \geq 0$, then $G$ is finitely presented and has a sub-cubic Dehn function.

Proof. Suppose $G$ acts properly, cocompactly, and by isometries on an $L_{\delta}$-space $X$. By the Švarc-Milnor Theorem [1, Proposition 8.19], $G$ is finitely generated and quasi-isometric to $X$. The result follows from Theorem 3.2.

\section{REFERENCES} 1999)

1. M. Bridson and A. Haefliger, Metric spaces of non-positive curvature (Springer-Verlag,

2. I. Chatterji, On property RD for certain discrete groups, Ph.D. Thesis, 2001. available at www.math.ohio-state.edu/ indira.

3. I. Chatterji and K. Ruane, Some geometric groups with rapid decay, Geometric and Functional Analysis 15 (2005), 311-339.

4. M. Elder, $L_{\delta}$ groups are almost convex and have a sub-cubic Dehn function, Algebraic and Geometric Topology 4 (2004), 23-29.

5. M. Gromov, Hyperbolic groups, in Essays in Group Theory (S. M. Gersten, editor), (Springer-Verlag, 1987), 75-263.

6. P. de la Harpe, Topics in geometric group theory (The University of Chicago Press, 2000).

7. M. Neumann and M. Shapiro, A short course in geometric group theory (Notes for the ANU Workshop, 1996). 